\title{
THREE-DIMENSIONAL MAGNETIC FIELD ANALYSIS OF A VARIABLY POLARIZING UNDULATOR
}

\author{
N. Nakamura, T. Shibuya, T. Koseki, H. Takaki \\ ISSP, University of Tokyo, Kashiwa, Chiba, Japan \\ Y. Kamiya, KEK, Tsukuba, Ibaraki, Japan
}

\begin{abstract}
The results of three-dimensional (3-D) magnetic field analysis of a variably polarizing undulator for the SuperSOR project are presented. In this analysis, the 3-D magnetic fields due to the magnet arrays of the undulator were calculated for three polarization modes and magnetic field errors due to iron beams supporting the magnet arrays were also estimated for the three modes. Furthermore effects of eddy currents of the undulator vacuum chamber were studied for $1 \mathrm{~Hz}$ switching of leftand right-handed circular polarization states that is achieved by mechanical driving of the magnet arrays.
\end{abstract}

\section{INTRODUCTION}

Super SOR (a new name of VSX) [1][2] is a thirdgeneration VUV and soft X-ray synchrotron light source and it will be constructed in a new campus of the University of Tokyo at Kashiwa (Kashiwa Campus), to which the ISSP have already moved out. Nine insertion devices will be installed in the ring. A probable candidate for insertion devices installed at the first phase is a variably polarizing undulator with a period length of 60 $\mathrm{mm}$ and a length of about $5 \mathrm{~m}(\mathrm{U} 60 \mathrm{~N})$. This undulator is an APPLE-2 type and has three polarization modes: horizontal, circular, vertical polarization modes. In addition, it can switch left- and right handed circular polarization states with a frequency of $1 \mathrm{~Hz}$ by mechanical driving of the magnet arrays. In this paper, the results of the 3-D magnetic field analysis of the variably polarizing undulator are reported.

\section{MAGNET STRUCTURE AND FIELD}

The schematic view of the U60N magnet structure is shown in Fig. 1. The undulator has four pure permanent magnet arrays and each array can be shifted longitudinally to produce various polarization states. The magnet block is made of a $\mathrm{NdFeB}$ material with a remanent field $\mathrm{B}_{\mathrm{r}}=$ $1.23 \mathrm{~T}$ and it is $40 \times 48 \mathrm{~mm}$ in horizontal( $\mathrm{x})$ and vertical(y) dimensions. The horizontal spacing between the left and right arrays is $1 \mathrm{~mm}$ and the minimum magnetic gap between the upper and lower arrays is 20 $\mathrm{mm}$.

The magnetic fields of U60N have been calculated by a computer code ELF/MAGIC[3], which is a solver for 3-D linear/nonlinear analysis of static and dynamic magnetic fields and based on IEM(Integral Element Method). On the central axis (z-axis), the horizontal and magnetic fields $\mathrm{B}_{\mathrm{x}}$ and $\mathrm{B}_{\mathrm{y}}$ sinusoidally change and are always $\pi / 2(15 \mathrm{~mm})$ apart in phase. Figure 2 shows the peak fields on the $\mathrm{z}$ axis as a function of the magnetic gap for three polarization modes: horizontal, circular, vertical polarization modes (HP, CP and VP modes). All the modes are achieved by adjusting the phase shift between two magnet arrays (upper left and lower right) and the other arrays (upper right and lower left). The phase shifts in HP and VP modes are 0 and $30 \mathrm{~mm}$ and the phase shift in CP mode depends on the gap (18.9 mm for the gap of $20 \mathrm{~mm}$ ). Figures 3 and 4 show horizontal and vertical distributions of the magnetic fields $\mathrm{B}_{\mathrm{x}}$ and $\mathrm{B}_{\mathrm{y}}$ for $\mathrm{CP}$ mode at the minimum gap. It is clearly found that these distributions are not well expressed with a single cosine or hyperbolic cosine function. The horizontal distribution of $B_{x}$ in the vicinity of the central axis can however be fitted with a cosine curve and the other field distributions with hyperbolic cosine curves. Effects of these magnetic fields on the electron beam in the Super-SOR ring are described in a separate paper [2].

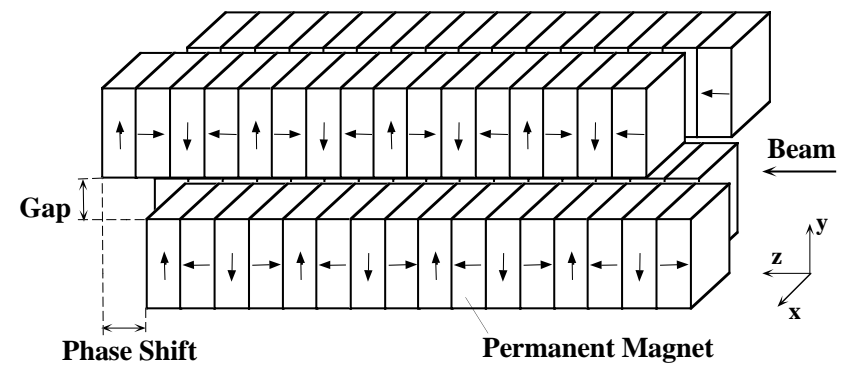

Figure 1: Schematic view of the magnet structure of the variably polarizing undulator $\mathrm{U} 60 \mathrm{~N}$.

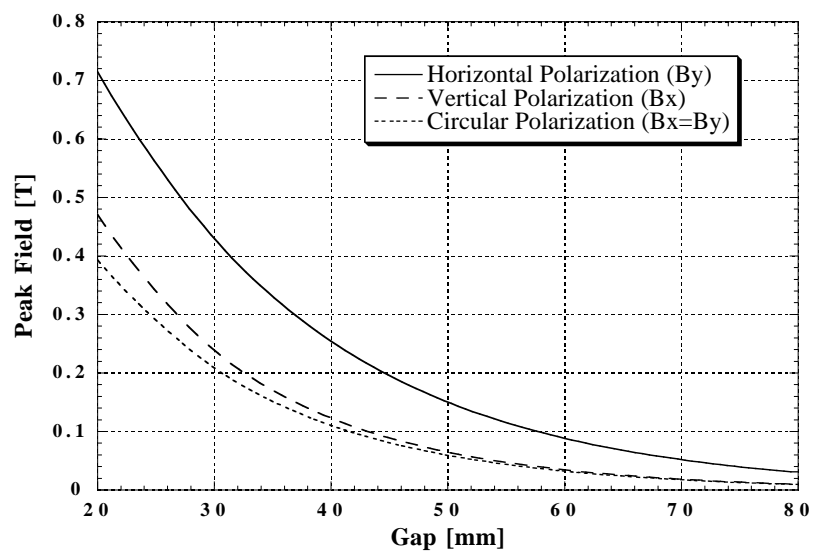

Figure 2: Peak field as a function of the gap of the variably polarizing undulator $\mathrm{U} 60 \mathrm{~N}$ for three polarization modes. 


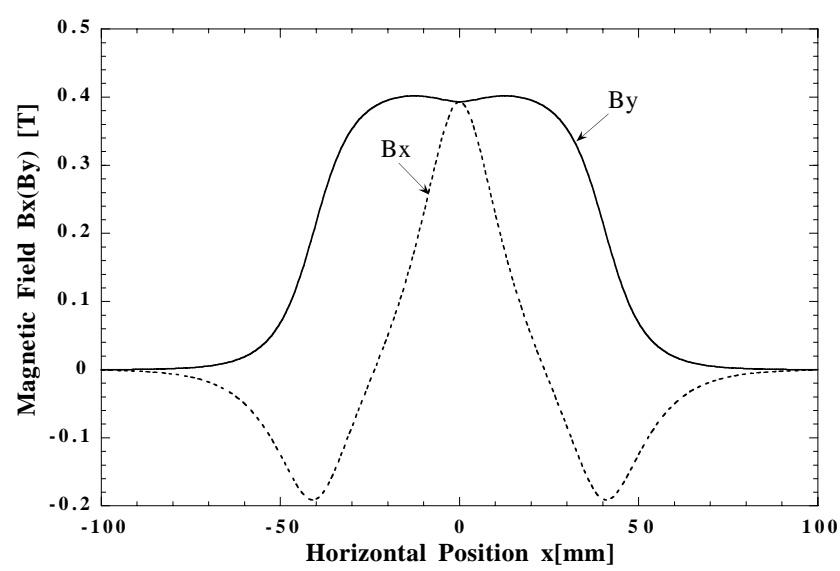

Figure 3: Horizontal distributions of the U60N magnetic fields $B_{x}(y=0 \mathrm{~mm}, z=15 \mathrm{~mm})$ and $B_{y}(y=z=0 \mathrm{~mm})$ for circular polarization mode.

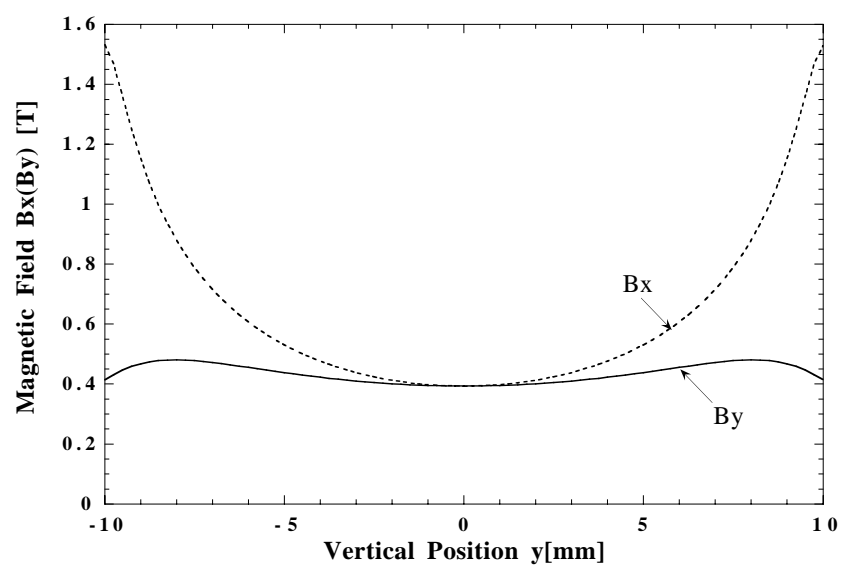

Figure 4: Vertical distributions of the U60N magnetic fields $B_{x}(x=0 \mathrm{~mm}, z=15 \mathrm{~mm}$,$) and B_{y}(x=z=0 \mathrm{~mm})$ for circular polarization mode.

\section{MAGNETIC FIELD ERRORS DUE TO SUPPORTING BEAMS}

The magnet arrays are fixed to supporting beams made of iron(SS400) through magnet holders and base plates made of stainless steel and moved both vertically and longitudinally with them. The supporting beams are the nearest ferromagnetic components to the magnet arrays and may deteriorate quality of the undulator field.

Magnetic field errors due to the supporting beams were calculated for three polarization modes by ELF/MAGIC. For the calculation, a model of U60N that has three periods with half-block ends was used and the permeability of the stainless steel components was assumed to be unity. In addition, the supporting beams were assumed to be iron bars that have the same length as the magnet arrays. Each bar has a cross section of $110 \mathrm{x}$ $30 \mathrm{~mm}$ and a distance of $52 \mathrm{~mm}$ from the magnet array fixed to it. Figure 5 show the distribution of the vertical field error along the z-axis at the minimum gap for $\mathrm{HP}$ mode. The field error gently changes along the z-axis compared to the undulator field itself and the magnitude of the error has a maximum value of about 3.5 Gauss at $\mathrm{z}=$ $0(0.5 \%$ of the central field). The distributions of the vertical fields for $\mathrm{CP}$ and VP modes are very similar in shape and magnitude to that for HP mode. For all the modes, the horizontal field error $\Delta \mathrm{B}_{\mathrm{x}}$ is less than 0.1 gauss and completely negligible. Figure 6 shows the vertical field error at $\mathrm{z}=0$ for HP mode as a function of the distance between the supporting beam and magnet array. It should be noted that the magnitude of the field error rapidly increases with decrease of the distance.

The additional calculation was carried out for other undulator models with 9 and 18 periods for HP mode to confirm that the field error does not much depend on the model. The result shows that the magnitudes of the vertical field errors are comparable with that of the 3period model and that the distribution curves are still gentle.

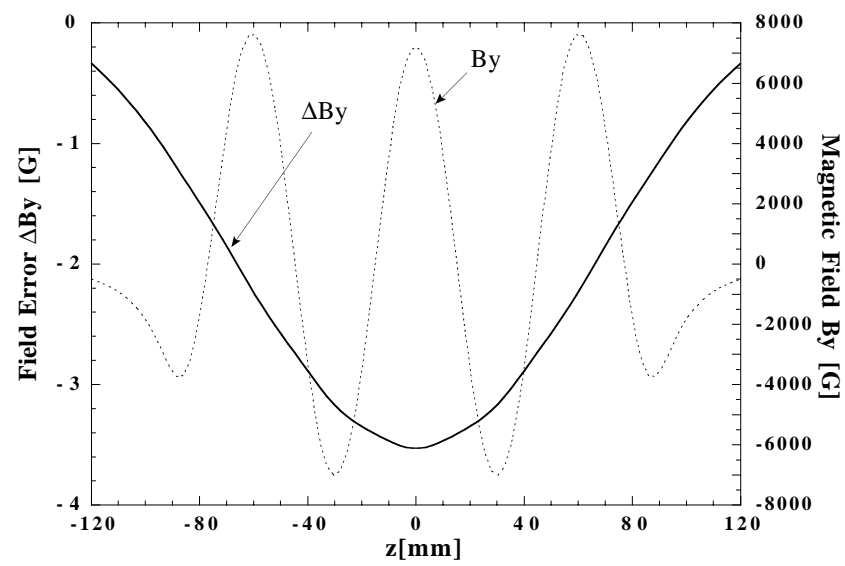

Figure 5: Longitudinal distribution of the vertical field error due to the supporting beams at the gap of $20 \mathrm{~mm}$ for HP mode. The vertical field without the supporting beams $B_{y}$ is also shown.

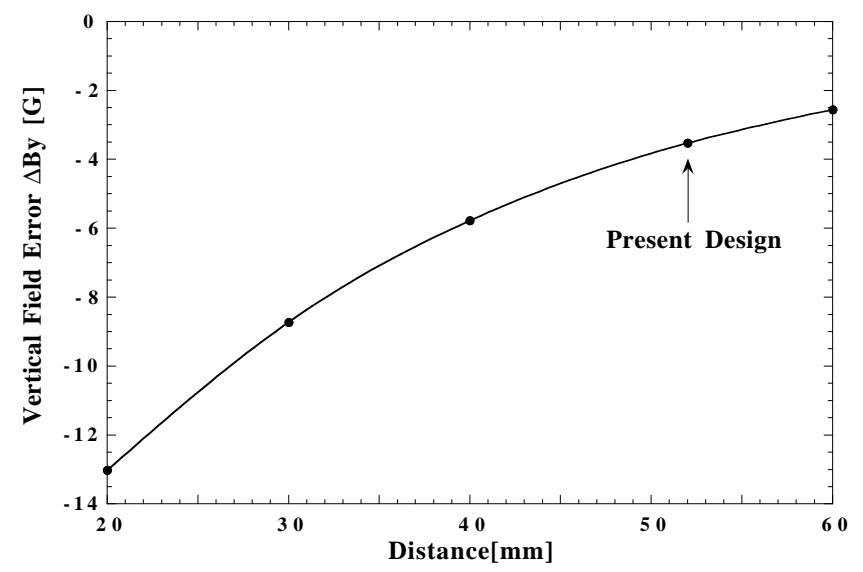

Figure 6: Vertical field error at the center $(\mathrm{z}=0)$ as a function of the distance between the supporting beam and magnet array at the gap of $20 \mathrm{~mm}$ for HP mode. 


\section{EFFECTS OF EDDY CURRENTS OF VACUUM CHAMBER}

For U60N, fast switching of left- and right-handed circular polarization states that is required for some experimental studies such as circular dichroism can be achieved by mechanical driving of the magnet arrays. The magnet motion causes eddy currents of the vacuum chamber and hence a dynamic error of the undulator field.

The magnetic field including effects of the eddy currents was calculated for the polarization-switching rate of $1 \mathrm{~Hz}$ by ELF/MAGIC. In the calculation, the 3-period model described in the previous section was used for the U60N undulator and the vacuum chamber was assumed to be a rectangular pipe with sizes of $60 \times 16 \times 450 \mathrm{~mm}$ (horizontal $\mathrm{x}$ vertical $\mathrm{x}$ longitudinal) and thickness of 1 $\mathrm{mm}$. Figure 7 shows the difference between the magnetic fields at the center $(x=y=z=0)$ with and without the vacuum chamber for three chamber materials, copper $(\mathrm{Cu})$, aluminium(Al) and stainless steel(SUS). The SUS chamber with the lowest conductivity has the smallest effect of the eddy currents on the undulator field. The field difference is less than 0.1 gauss. For this reason, SUS will be adopted as the U60N chamber. However, since the SUS chamber has high resistive-wall impedance causing transverse coupled-bunch instabilities, the inner surface of the chamber will fully or partially be covered by a copper coating with thickness of $0.1-0.2 \mathrm{~mm}$ to reduce the resistive-wall impedance [3]. The effect of eddy currents of the $0.1-\mathrm{mm}$ thick copper coating was also calculated in a similar way and its result is shown in Fig. 8 . In the figure, the partial coating means that copper is coated from $\mathrm{x}=-1 \mathrm{~cm}$ to $\mathrm{x}=+1 \mathrm{~cm}$ on the upper and lower inner surfaces. The effect of the copper coating on the undulator field is not serious, though it is larger than that of the SUS chamber itself.

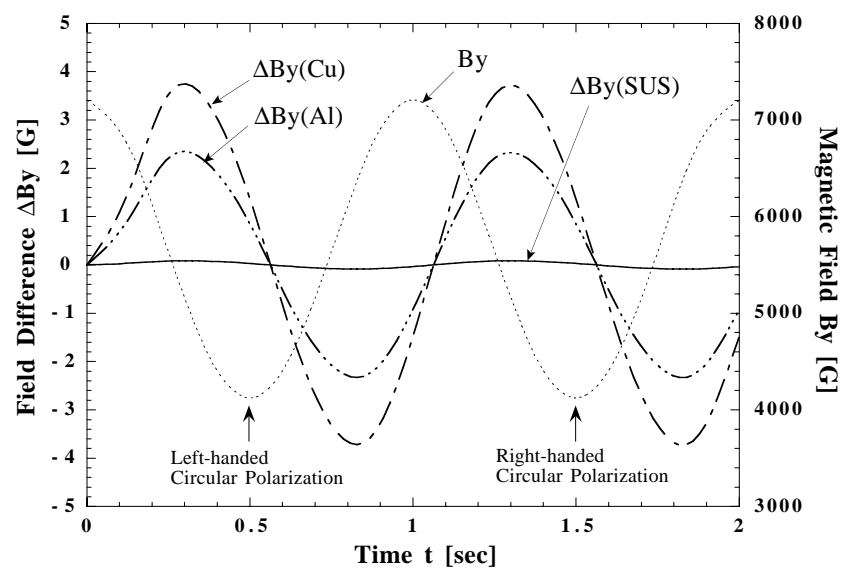

Figure 7: Difference $\Delta \mathrm{B}_{\mathrm{y}}$ between the vertical fields at the center with and without the vacuum chambers made of $\mathrm{Cu}, \mathrm{Al}$ and SUS for the initial 2 seconds of the polarization switching due to mechanical driving of the magnet arrays. The vertical field without the chamber $\mathrm{B}_{\mathrm{y}}$ is also shown.

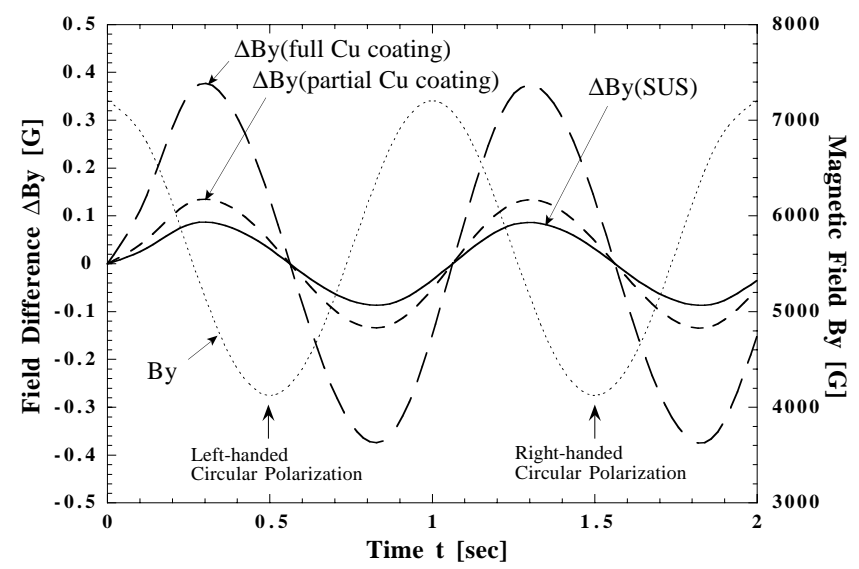

Figure 8: Difference between the vertical fields with and without the full or partial $\mathrm{Cu}$ coating. For the partial coating, the coating is confined to the horizontal position range of $-1 \mathrm{~cm}$ to $+1 \mathrm{~cm}$ on the upper and lower inner surfaces. For comparison, the field difference of the SUS chamber itself is shown.

\section{CONCLUSION}

The variably polarizing undulator (U60N) was designed and its 3-D magnetic fields were calculated for three polarization modes. The calculated peak fields and the field distributions are used to study characteristics of the undulator radiation and effects on the electron beam. The calculation result also shows that the field errors due to the iron supporting beams are tolerable for the distance of $52 \mathrm{~mm}$ from the magnet arrays. For the $1 \mathrm{~Hz}$ polarization switching due to mechanical driving of the magnet arrays, SUS is the best material for the vacuum chamber, because it has much less eddy currents than $\mathrm{Cu}$ and $\mathrm{Al}$. The copper coating that reduces the resistive-wall impedance of the SUS chamber is sufficiently thin to have small eddy currents. The 3-D static and dynamic field analysis is very important and useful for the undulator design.

\section{REFERENCES}

[1] N. Nakamura, "New Design and Developments of the VSX Light Source", Proc. of EPAC2000, Vienna, 2000, p.669.

[2] K. Harada, M. Satoh, H. Takaki, T. Koseki, N. Nakamura and Y. Kamiya, "The Effects of the Insertion Devices at the Super SOR Light Source", these proceedings.

[3] http://www.iijnet.or.jp/ELFMAGIC/index.html

[4] N. Nakamura, Y. Kamiya, T. Koseki, Y. Takiyama, K. Shinoe, M. Fujisawa, Y. Hori, S. Mandai and M. Oishi, "Reduction in Resistive-Wall Impedance of Insertion-Device Vacuum Chamber By Copper Coating", Proc. of EPAC'98, Stockholm, 1998, p.984. 